\title{
Proceedings of the 131st Semon Club, Otolaryngology Department, Guy's and St Thomas' NHS Foundation Trust, London, UK, 26 May 2006
}

Chairman: Miss E Chevretton, Consultant ENT Surgeon, Guy's and St Thomas' NHS Foundation Trust, London Pathologists: Professor L Michaels and Dr A Sandison, University College London Radiologist: Dr E Woo, Specialist Registrar, Guy's and St Thomas' NHS Foundation Trust, London Secretary: Mr M Black, Specialist Registrar, Guy's and St Thomas' NHS Foundation Trust, London A prize was awarded for the best presentation of the meeting.

\section{Otology and skull base session \\ Chairman: Mr A Fitzgerald-O’Connor}

\section{Benign necrotizing otitis externa: a rare, dramatic} presentation

Y Ramakrishnan, B McMonagle, M J Gleeson

From the Guy's \& St Thomas' Foundation NHS Trust, London, UK

\section{Case report}

A 59-year-old woman presented with an extensive necrotizing ulcer involving her left temporal bone. This had developed over a period of six months, during which time she had experienced persistent otorrhoea, pain, nausea and a tendency to fall to the left side. In the months preceding her referral, she had undergone a modified radical mastoidectomy and several meatoplasties. Her past medical history included depression and alcohol abuse.

Examination revealed a large area of exposed temporal bone, with the temporomandibular joint visible. The conchal cartilage was necrotic and the mastoid cavity infected. Both computed tomography (CT) and magnetic resonance imaging were performed, following which a diagnosis of benign necrotizing otitis externa was made.

The necrotic tissue was debrided and a subtotal petrosectomy performed using a vertical rectus abdominis myocutaneous flap for reconstruction. The patient made an uncomplicated recovery.

\section{Discussion}

The chairman discussed the rarity of this condition. As the symptoms are not dissimilar to malignant otitis externa and temporal bone carcinoma, both histology and appropriate imaging (bone scan and CT) will be helpful in guiding diagnosis. Professor Michaels advised that the aetiology of this condition is unclear, and he confirmed that surgical clearance was the treatment of choice.

\section{How to discriminate external auditory canal cholesteatoma from keratosis obturans?}

T Mirza, S C L Leong, D K Selvadurai

From St George's Hospital, London, UK

\section{Case report}

A 46-year-old woman was referred with a long history of what was perceived by her general practitioner to be right otitis externa. On examination, the tympanic membrane appeared normal, with no signs of middle-ear pathology, although a 'pit-like' lesion was noted in the external auditory canal (EAC). A computed tomography scan showed a bone-destroying lesion in the inferior aspect of the EAC, extending to the temporomandibular joint. The lesion was excised and the canal defect repaired as a meatoplasty. Histology showed keratinizing squamous epithelium consistent with a diagnosis of cholesteatoma.

\section{Discussion}

External auditory canal cholesteatoma is a rare condition that needs to be distinguished from cholesteatoma of the middle-ear space and from keratosis obturans. Professor Michaels explained that, unlike keratosis obturans, there is focal ulceration and bone erosion in EAC cholesteatoma. He explained that the aetiology of EAC cholesteatoma is not fully understood and that the condition may arise following trauma, with the ensuing inflammatory response proceeding to an osteitis with bony destruction. $\mathrm{He}$ also postulated that the epithelium of the inferior auditory canal may migrate at a slower rate, causing accumulation of epithelial debris and inflammation. $\mathrm{Mr}$ Fitzgerald-O'Connor suggested that an aspiring trainee consider further studies on epithelial migration.

\section{An unusual tumour of the ear}

F Amen, D Roy

From St John's Hospital, Chelmsford, UK

\section{Case report}

A 32-year-old Asian lady presented with a three-month history of tinnitus and intermittent right ear infections. Examination revealed a cyst arising from the posterior wall of the external auditory canal, which was excised under local anaesthesia and found to extend into the mastoid. A magnetic resonance imaging (MRI) scan showed a well circumscribed, $2.6 \times 1.4 \times 1.2 \mathrm{~cm}$ mass in the lateral aspect of the right middle-ear cavity, with extension through the tympanic membrane.

A mastoidectomy was performed, revealing a large mass in the mastoid cavity eroding the posterior canal wall. The posterior canal wall was reconstructed using tragal cartilage and periosteal and skin flaps. The vertical segment of the facial nerve was exposed. Histology was suggestive of a rare ceruminous adenocarcinoma. Two weeks after the operation, the patient developed a right-sided, lower motor neuron facial palsy that resolved after removal of the bismuth iodoform paraffin paste pack and administration of prednisolone. The patient was free of recurrence at seven months' follow up.

\section{Discussion}

Mr Fitzgerald-O'Connor was of the opinion that the ear should be re-explored at six months to check for 
recurrence. Mr David Howard (RNTNE) recommended close, long term follow up, including annual MRI scanning, to check for local recurrence.

\section{An unusual swelling in the external auditory canal}

G Ninkovic, D Bray, N Saunders

From the Royal Sussex County Hospital, Brighton, UK

\section{Case report}

A 49-year-old man presented with a sensation of blockage, intermittent swelling and hearing loss in the left ear. Otoscopy confirmed a large, cystic, postero-superior swelling causing occlusion of the external auditory canal (EAC). The overlying skin was normal. Computed tomography of the temporal bone confirmed a soft tissue swelling in the left EAC. Two millilitres of turbid fluid was aspirated and a keratin plug deep to the swelling was removed. Analysis of the aspirate showed no organisms or cells. The patient's symptoms recurred six months later, and excision of the lesion via a post-auricular approach was performed. Histology revealed a benign apocrine hydrocystoma.

\section{Discussion}

An apocrine hydrocystoma is an intradermal, blue, usually solitary cyst of apocrine origin commonly seen on the cheeks, eyelids, scalp and chest. It presents in middle age and has equal sex prevalence. The prevalence of apocrine hydrocystoma in the ear is unknown. Glandular tumours in the ear used to be known as ceruminomas until 1972, when they were further subclassified by Wetli.

Apocrine hydrocystomas can mimic basal cell carcinoma clinically, and they are often confused histologically with adenocarcinoma. As a result, some patients have in the past been subjected to extensive surgical resection and radiotherapy.

\section{Pulsatile tinnitus: the importance of diagnosis and treatment}

M-C Jaberoo, A Prasai, A Juratli

From the Leicester Royal Infirmary, Leicester, UK

\section{Case report}

A 62-year-old woman presented with a one-month history of right-sided, pulsatile tinnitus. The patient noticed that the tinnitus stopped when she compressed the right postauricular area. Otoscopy showed no abnormality. Examination of the right post-auricular area revealed abnormal pulsation and, on auscultation, a vascular murmur. An audiogram showed bilateral high frequency hearing loss. Tympanograms were within normal limits.

Magnetic resonance imaging angiography demonstrated a vascular malformation in the right temporal bone supplied from the right external carotid artery, with a fistula communicating with the right transverse/sigmoid sinus. The patient was referred to the regional neurosurgical unit for conventional digital subtraction angiography. Embolization was discussed; however, as the tinnitus had resolved the patient declined intervention.

\section{Discussion}

Pulsatile tinnitus associated with normal findings on otoscopy can pose a diagnostic dilemma. It may represent a serious vascular malformation. Diagnostic evaluation should include a detailed history, otoneurological examination, audiological assessment and relevant imaging. Mr Fitzgerald-O'Connor commented that if a retrotympanic mass was seen on otoscopy, a myringotomy should not be undertaken without relevant pre-operative imaging.

\section{Paediatric session}

Chairman: Mr G Morrison

An unusual presentation of congenital cholesteatoma

C T Huins, S Abramovich

From St Mary's Hospital, London, UK

Case report

A previously otologically asymptomatic, three-year-old child presented to his general practitioner (GP) with an upper respiratory tract infection. During routine examination, the GP noticed an unusual appearance of the left tympanic membrane and referred the child for specialist opinion.

Our otoscopic examination revealed a white mass medial to the antero-superior aspect of an intact left tympanic membrane. Audiometry and tympanometry were normal.

Subsequent computerized tomography scanning of the temporal bones revealed a well defined, lobulated, $5 \mathrm{~mm}$ focus of soft tissue in the mesotympanum, medially abutting the processus cochleaformis.

A left atticotomy and tympanoplasty were performed. The white, well circumscribed lesion had not eroded any of the ossicles and was completely removed. Histology confirmed cholesteatoma.

\section{Discussion}

A thorough examination by the patient's diligent GP resulted in this potentially destructive lesion being discovered early, limiting operative intervention and preserving hearing.

Professor Michaels commented that the higher incidence of congenital cholesteatoma in the USA might be due to experienced, paediatrically orientated GPs performing baby checks. He also proposed that an unknown proportion of acquired cholesteatomas might have actually originated as congenital cholesteatomas. This case reiterates the importance of simple otoscopy in routine ENT examination.

\section{An unusual presentation of retropharyngeal abscess}

R S Natt, C Georgalas, N S Tolley From St Mary's Hospital, London, UK

\section{Case report}

An 18-month-old boy was admitted by the paediatric team for intravenous antibiotics following a three-day history of a sore throat and a non-fluctuant left neck swelling. An ultrasound demonstrated cervical lymphadenopathy. The child clinically deteriorated, and an ultrasound 48 hours later displayed gas and a fluid collection in the neck. A computed tomography of the neck and thorax demonstrated a retropharyngeal abscess extending from the skull base to the carina.

The patient underwent external drainage of the retropharyngeal abscess and an urgent panendoscopy, which revealed a perforation of the hypopharynx. This was left to heal by secondary repair and treated as a pharyngocutaneous fistula. The patient's older sibling later admitted to toothbrush-related pharyngeal trauma three days prior to the patient's admission, explaining the perforation.

\section{Discussion}

The commonest cause of retropharyngeal abscess in children is suppuration of retropharyngeal lymph nodes secondary to upper respiratory tract infection. In this particular case, the cause was secondary to hypopharyngeal perforation and could have been missed, as there was no 
initial history suggesting this. It is imperative to take a detailed history and to exclude a traumatic cause in cases of paediatric retropharyngeal abscess which cannot be explained by an infective cause. A non-accidental injury must always be considered.

\section{Abnormal course of internal carotid artery in a child with fragile $X$ syndrome}

V S Singh, P Priyadarshini, S Thorogood

From the East Surrey Hospital, Redhill, UK

\section{Case report}

An eight-year-old boy with fragile $\mathrm{X}$ syndrome presented with recurrent otitis media with effusion and sleep apnoea syndrome. He could not tolerate continuous positive airway pressure and was booked for adenotonsillectomy and grommet insertion.

During the procedure, the surgeon found abnormal pulsations in the nasopharynx. Adenoidectomy was therefore abandoned, although tonsillectomy with insertion of grommets was performed. A subsequent magnetic resonance imaging scan revealed the right internal carotid artery to be very close to the adenoids.

\section{Discussion}

Fragile $\mathrm{X}$ syndrome is one of the most common causes of mental retardation. It mostly affects males (1:4000) and involves mutation at the fragile $\mathrm{X}$ mental retardation-1 site of the $\mathrm{X}$ chromosome. The child usually has a large body size, prominent jaw, suffers from recurrent otitis media with effusion and has hyperactive behaviour.

Mr Morrison was of the opinion that, in patients in whom pulsation is noted in the nasopharynx, adenoidectomy could be performed under direct vision using a microdebrider. The 'take-home message' from Mr David Howard (RNTNE) was that it was absolutely essential to examine the nasopharynx peri-operatively before performing adenoidectomy.

\section{An unusual cause of stridor}

W R Perera, D Gillett, A Rachmanidou

From the University Hospital Lewisham, London, UK

\section{Case report}

A 73-year-old woman presented with acute onset, biphasic stridor, hoarseness and laryngeal discomfort. She had previously undergone a right upper lobectomy in October 2004 for non-small cell lung carcinoma. A silicone Y stent was sited in August 2005, as she subsequently developed tracheal narrowing due to extrinsic compression by recurrent mediastinal disease.

A chest X-ray showed proximal stent migration, causing the two distal lumen (the bifurcation of which was intended to lie on the carina) to become occluded, producing stridor and desaturation. In theatre, the stent was removed using bronchoscopy forceps whilst maintaining oxygenation via jet ventilation, under general anaesthesia. Subsequent tracheoscopy showed no abnormalities. The patient remained well post-operatively.

\section{Discussion}

Silicone stents are the most commonly used stent for palliation of central obstructive lesions. Stent migration is a known complication (9.5-24 per cent) and is commoner with silicone stents. This is due to the expansile characteristics of metallic stents, providing a tighter fit, as opposed to silicone, which expands to reach a preformed shape.

\section{Stridor: double trouble}

R K Sharma, D Jiang, G A J Morrison

From the Guy's \& St Thomas' Foundation NHS Trust, London, UK

\section{Case report}

A 10-day-old male infant presented with intermittent stridor and neck swelling. Examination confirmed a diffuse, cystic, fluctuant swelling in the right neck. A microlaryngobronchoscopy (MLB) revealed a posterior pharyngeal wall swelling extrinsically compressing the larynx. A computed tomography scan confirmed a $6 \times 6 \times 2.5 \mathrm{~cm}$ cystic mass in the right neck crossing the midline. An almost complete excision of the hygroma was performed, preserving the phrenic, hypoglossal and vagus nerves. The patient was successfully extubated and discharged home.

Three months after the procedure, the patient developed a recurrence of stridor. A repeat MLB revealed a small remnant of cystic hygroma behind the tonsil. The infant's larynx appeared to show features consistent with laryngomalacia. An aryepiglottoplasty was performed and the symptoms resolved.

\section{Discussion}

A fast-growing cystic hygroma compromising the airway requires early surgical excision. However, complete excision at the cost of function is not justified. Laryngomalacia is the commonest cause of stridor in infants; this worsens with age and is likely to be overlooked in the presence of more prominent pathology, such as the cystic hygroma presented here. It is important to reassess the airway and to ensure appropriate management in the presence of ongoing symptoms.

\section{Head and neck session}

Chairman: Mr R Simo

\section{An unusual presentation of a parotid tumour}

A Sanei-Moghaddam, S C L Leong, T Odutoye

From St George's Hospital, London, UK

Case report

A 52-year-old man presented with a six-month history of painless, bilateral swelling of the parotid gland. His facial nerve function was intact. Magnetic resonance imaging showed multi-centric lesions with increased attenuation in both parotid glands, whilst fine needle aspiration cytology was suggestive of acinic cell carcinoma or oncocytoma. The patient underwent bilateral total parotidectomy and level one to three neck dissection, followed by radiotherapy.

\section{Discussion}

Acinic cell carcinoma is a rare and slow-growing tumour of the parotid gland. Professor Michaels explained that the histological appearance of clear cells may be indicative of metastatic renal cell carcinoma, although he concluded that this was a remote possibility in this case as the specimen was CD-10 negative. Furthermore, ultrasound of the kidneys was normal. The role of positron emission tomography scanning was also discussed as an appropriate investigation. This case highlighted two distinct schools of thought regarding patient management. Mr David Howard (RNTNE) suggested that, due to the unknown natural course of the tumour, aggressive management of these patients may be difficult to justify. He suggested bilateral parotidectomy and close follow up as an adequate 
alternative to the treatment recommended by the multidisciplinary team meeting at St George's Hospital.

\section{Recurrent self-healing 'squamous cell carcinoma' of the skin}

D E Whitehead, E Nilssen

From the Queen Alexandra Hospital, Portsmouth, UK

\section{Case report}

A 71-year-old woman from Ayrshire in Scotland presented with a history of recurrent facial kerato-acanthomas from 1959 onwards. The condition worsened during the summer and the lesions increased in number and frequency with age. When left untreated, the lesions spontaneously resolved, leaving depressed scars.

Histology showed marked hyperkeratosis and parakeratosis, with mild architectural and cytological atypia. The appearances were those of an actinic keratosis; however, well differentiated squamous cell carcinoma could not initially be excluded. A diagnosis of multiple self-healing squamous epithelioma syndrome was finally made in 1999 after a complete family history was obtained.

This rare, autosomal dominant, hereditary condition was first described by a general practitioner, Dr Ferguson Smith, in 1934. It is probable that all the Scottish cases were derived from a single mutation on chromosome 9 , which occurred before 1790 .

\section{Discussion}

Dr Sandison confirmed that, without the medical history, a histological diagnosis of well differentiated squamous cell carcinoma would have been made. Mr David Howard (RNTNE) agreed that detailed history-taking was vital in order to identify affected individuals. A careful history should aid identification and provide guidance in order to avoid aggressive and unnecessary treatment for this rare, hereditary, self-limiting condition.

\section{Persistent and progressive dysphagia: an interesting case report}

N Donnelly, S K Balasubramaniam, D B Mitchell From the Kent \& Canterbury Hospital, Canterbury, UK

\section{Case report}

A 32-year-old woman with mild dysphagia for 15 years presented with worsening of symptoms. She was an asthmatic and had no history of gastroesophageal reflux disease or anaemia. Her ENT examination was normal. Oesophagoscopy revealed a friable web below the cricopharyngeus, which was dilated and biopsied. Biopsy findings were indicative of eosinophilic oesophagitis.

\section{Discussion}

This is an increasingly recognized condition, characterized by eosinophilic infiltration of the oesophageal mucosa. Clinical signs and symptoms may be indistinguishable from gastroesophageal reflux disease. Therapeutic recommendations include systemic or topical corticosteroids, leukotrine receptor antagonists and hypo-allergic diets. The condition does not respond to standard gastroesophageal reflux disease treatment.

Mr David Howard (RNTNE) recommended that all patients with swallowing difficulties be primarily investigated with video fluoroscopy by specialized speech therapists. It was recognized that eosinophilic oesophagitis may be under-diagnosed in otolaryngological practice. Dilatation of strictures in these patients should be avoided.
Liposarcomatous transformation of a pre-existing cervical lipoma

K Saravanan, A Belloso, M Small

From the Royal Preston Hospital, Preston, UK

Winner of the prize for best presentation of the meeting.

\section{Case report}

A 58-year-old man presented with a progressively enlarging, left-sided, posterior triangle cervical mass. Eleven years prior, the patient had had a tru-cut biopsy, confirming lipoma, at the same site. Magnetic resonance imaging showed the mass extending into the cervical spinal canal at $\mathrm{C} 1 / 2$. Excision of the mass was accompanied by a type two modified neck dissection. Pathologic examination revealed the presence of a dedifferentiated liposarcoma with well differentiated, lipoma-like and liposarcomatous areas. The patient had a recurrence of the liposarcoma, which was excised again. The patient was symptom free at one year follow up.

\section{Discussion}

Mr David Howard (RNTNE) held the view that liposarcomatous transformation from a previously diagnosed head and neck lipoma is not known to occur. In the presented case, the inadequacy of the sample from the original lipoma could not support the possibility of liposarcomatous transformation. It must be concluded that this case was indeed a liposarcoma from the outset, co-existent within a lipoma. Any lipoma presenting deep in the head and neck region should prompt suspicion of a coexistent liposarcoma. Thorough clearance of the lesion and good pathological examination are vital to diagnosis of the condition

\section{Marjolin's ulcer in a pharyngeal pouch}

N Ibery, D Gillett, T Jacob

From the University Hospital Lewisham, London, UK

\section{Case report}

A 90-year-old woman was transferred from a local district general hospital with a history of worsening dysphagia. In spite of her age, she had relatively little co-morbidity. Three days prior to presentation, the patient had undergone an oesophago-gastroduodenoscopy which had identified a pharyngeal pouch but no other abnormality. This pouch had been initially diagnosed over 30 years ago, but the patient had not received any definitive treatment.

A pan-endoscopy was performed with a view to endoscopic stapling if appropriate; however, intra-operatively, a large tumour was found arising from the pouch. The biopsies demonstrated a poorly differentiated, keratinizing, squamous cell carcinoma. Computed tomography confirmed metastatic spread, and palliative treatment was recommended.

\section{Discussion}

It has been hypothesized that carcinoma arising in a pharyngeal pouch develops in much the same way as squamous cell carcinoma in the presence of chronic irritation or nonhealing ulceration (i.e. Marjolin's ulcer). If not detected early, the pharyngeal pouch tumour may spread rapidly. We propose that careful consideration should be given to resection of long-standing pouches that have been neglected for several years, as these have a high risk of malignancy. 
Rhinology session

Chairman: Mr N Singh

\section{An unusual cause of a maxillary mass}

M Lim, S Lew-Gor, V J Lund

From the Royal National Throat, Nose and Ear Hospital, London, UK

\section{Case report}

A 56-year-old woman presented with a six-month history of right-sided nasal blockage, swelling of the right side of the face and blood-stained nasal discharge. Examination revealed right eye proptosis, with bulging of the right lateral nasal wall on endoscopy.

A computed tomography scan showed an expanded, homogenously opaque right maxilla, with elevation of the floor of the orbit and extension into the nasal cavity.

Endoscopic sinus surgery was performed, revealing a haemorrhagic mass in the maxillary sinus with no obvious point of origin. A large middle meatal antrostomy was fashioned. The mass was removed completely, leaving the mucosa intact. Histological analysis of the mass showed it to be simply a blood clot. Further questioning did not reveal any history of a bleeding disorder or previous trauma.

\section{Discussion}

Dr Woo felt that, based on the appearance of the scan, the most likely diagnosis would have been a mucocele. Professor Michaels concurred on the basis of the histological findings. Spontaneous, organized haematoma of the maxillary sinus is rarely reported, with only 12 published cases. The lesion is clinically significant because, although histologically benign, it may present in a progressive manner. The treatment of choice is surgical evacuation.

\section{Endoscopic orbital decompression for 'popeyes'}

S A Hannan, A A Roy, L Badia

From the Royal National Throat, Nose and Ear Hospital, London, UK

\section{Case report}

A fit, 70-year-old man presented to his ophthalmologist with a long history of his eyes 'popping out', especially overnight. He could always pop them back in. Examination confirmed mild bilateral proptosis. Orbital palpation produced reducible luxation. Thyroid function tests were normal. Computed tomography confirmed bilateral proptosis with shallow orbits. The patient was referred for novel consideration of endoscopic orbital decompression to manage his 'popeyes'.

The patient subsequently underwent elective surgery and had an uncomplicated post-operative course. Over a year's follow up, the patient demonstrated improved ocular cosmesis, no ocular motility problems and no further episodes of spontaneous globe luxation.

\section{Discussion}

Spontaneous globe luxation is rare. Congenitally shallow orbits and lax orbital septa conspire to allow forward displacement of the globes. If not reduced, there is the risk of optic nerve strangulation and blindness. Conventional management concentrates on manual reduction, ocular surface lubrication and eyelid procedures but does little to address proptosis and luxation.

Endoscopic orbital decompression is established treatment for inflammatory and compressive orbital diseases, such as dysthyroid ophthalmopathy, in which proptosis is significant. We report the innovative use of such surgery as successful treatment for spontaneous globe luxation. We encourage other ophthalmologists and otorhinolaryngologists to consider endoscopic orbital decompression for 'popeyes'.

\section{Sudden onset nasal speech}

S Khan, J Rimmer, J Almeyda

From the West Middlesex Hospital, London, UK

\section{Case report}

A 25-year-old man presented with the recent onset of slurred, nasal speech. He was a smoker and admitted to using cocaine. Examination revealed a saddle nose deformity, extensive crusting, septal destruction, erosion of the soft and hard palate with loss of the uvula, and posterior pharyngeal wall necrosis.

Serological tests were positive for perinuclear antineutrophil cytoplasmic antibody (pANCA) but negative for circulating anti-neutrophil cytoplasmic antibody (cANCA). Computed tomographic scanning demonstrated destruction of the nasal septum, medial maxillary sinus walls and palate. Biopsies were consistent with cocaine-induced destruction; however, further investigation was recommended to exclude Wegener's granulomatosis.

\section{Discussion}

Raised ANCA levels may be seen in patients with cocaine-induced, destructive midline lesions. Wegener's granulomatosis can present similarly, with cANCA being positive in 98 per cent and pANCA positive in 5-20 per cent of cases. Professor Michaels held the opinion that cocaine abuse was the underlying cause in the presented case.

This case highlights a diagnostic dilemma: malignancy, inflammatory and vasculitic disorders. Miss Chevretton and $\mathrm{Mr}$ Singh felt it important to clarify that raised ANCA levels were not exclusive to vasculitis. Following general discussion, participants agreed that drug screening for cocaine use may be useful to avoid misdiagnosis, and also that cessation of drug usage is required to ensure the long-term success of reconstruction.

\section{An unusual case of epistaxis}

A Masood, S Ray, O P Chawla

From the Luton and Dunstable Hospital, Luton, UK

\section{Case report}

A 34-year-old Asian man presented to the ENT clinic with right nasal blockage and recurrent epistaxis. A pink, polypoidal mass was seen arising from the right inferior turbinate. Excision biopsy revealed hyperplastic epithelium and squamous metaplasia, along with the presence of large numbers of sporangia and endospores typical of rhinosporidiosis. Further management included long-term dapsone (4,4-diaminodiphenyl sulphone). The patient underwent multiple repeat excisions. At the time of writing, he had been symptom free for two years.

\section{Discussion}

Rhinosporidiosis is caused by Rhinosporidium seeberi, an aquatic, protistan parasite. Professor Michaels noted that the disease spreads through the release of endospores in water. It is an infective but not a contagious disease. Susceptibility of individuals to infection is still a matter of debate. Although described over 100 years ago, several doubts persist due to the lack of a method to culture $R$. seeberi in vitro and the failure to induce rhinosporidiosis in experimental animals. 
Rhinosporidiosis is very rare in the UK and Europe. It is mainly seen in the Indian sub-continent. In view of the large migrant population in the $\mathrm{UK}$, rhinosporidiosis should be kept in mind in the differential diagnosis of bleeding nasal polyps.

\section{Nasal teeth}

M Eldardiri, R Kaddour, H S Kaddour

From the Harold Wood Hospital, Romford, UK

\section{Case report}

A 60-year-old Caucasian man presented with chronic nasal blockage, hyposmia and intermittent facial pain, not responding to medical therapy. Nasendoscopy revealed bilateral, diffuse, grade three nasal polyposis, a deviated nasal septum and a possible foreign body in the right nasal cavity.

Computed tomography showed a radiopaque lesion in the floor of the right nasal cavity. The patient underwent endoscopic ethmoidectomy and polypectomy, during which a nasal tooth was noted in the right nasal floor, which was extracted endoscopically. He made an uneventful post-operative recovery.

\section{Discussion}

Nasal teeth are very rare, with only 50 cases reported in the literature. Nasal teeth can be extra-numerary or ectopic. The aetiology is unknown. However, several predisposing factors have been suggested, including genetic predisposition, infection, dental trauma and crowded dentition. The differential diagnosis of nasal teeth includes: rhinolith, foreign body, granulomatosis, and benign or malignant tumours.

Surgical extraction of nasal teeth is recommended in symptomatic cases. Twenty-five per cent of reported cases have been associated with chronic rhinosinusitis. In our case, the nasal tooth was associated with nasal polyposis. 